\title{
Modelling reactive diffusion in clays with two-phase- informed pore networks
}

4

\section{Abstract}

10 Development of pore network models for reactive diffusion of various

\author{
Qingrong Xiong*, Andrey P Jivkov, Syed M Ahmad \\ Research Centre for Radwaste \& Decommissioning and \\ Modelling \& Simulation Centre, Dalton Nuclear Institute, \\ The University of Manchester, UK.
} measured pore-space and solid-phase characteristics. This way incomplete geometrical and topological information for a given pore system can be balanced by known solid-phase properties. Opalinus Clay (OPA) is selected to demonstrate the application and validate the proposed model. OPA is modelled as anisotropic porous medium, reflecting preferential orientation of meso-porosity along clay bedding direction. Bulk diffusivities of various species $\left(\mathrm{HTO}, \mathrm{Cl}^{-}\right.$and $\left.I\right)$ are calculated to investigate the effects of pore structure, anion exclusion and sorption. Sorption is simulated by changing the pore space, which is more realistic compared to existing reactive transport models with assumed constant pore geometry. Anion exclusion effects are simulated by introducing diffuse double layer (DDL) in the model. Results agree well with experimentally measured diffusion coefficients for transport parallel and perpendicular to the bedding direction. The proposed model is applicable to a large class of geo-materials and suitable for linking to lattice models for deformation and damage.

\section{Introduction}

A thorough understanding of the porous media mass transport is of prime importance in various science and engineering applications, e.g., in radioactive waste disposal $[1,2]$, in remediation of contaminated groundwater [3], in tracer studies in oil recovery [4]. The transport properties such as permeability and diffusivity vary with pore space changes due to various mechanical, chemical, physical and thermal processes. These processes can be simulated and analysed by developing appropriate physical and microstructure-informed models. Such models are required to be able to predict measurable transport properties at a macro-scale (considering engineering and geological aspects of the formation) from measurable pore space characteristics such as pore shapes, pore size distribution. 
1 Pore network models (PNMs) provide a suitable representation of mutable

2 pore space structures. In these models, the pore space is approximated by a

3 set of sites and a set of bonds connecting some of the sites [5, 6]. PNMs

4 have to reflect the basic properties of porous media, such as shape and size

5 distribution of pores and throats, as well as the pore coordination spectrum,

6 i.e. the percentages of pores coordinated by different number of throats $[7$,

7 8]. These basic properties can be obtained in structures with distinguishable

8 pores and pore throats. However, in materials such as Opalinus Clay (OPA),

9 the pore space is dominated by pores smaller than $50 \mathrm{~nm}[9,10]$, and due to

10 the limitation resolution of current techniques the pore connectivity data

11 cannot be extracted [11]. Hence, for such cases with limited pore space

12 structure information, a different approach is required to construct effective

13 pore networks. Previous approaches to tackle such incomplete pore space

14 information, including predefined connectivity to calculate length scale

15 [12], and variable length scale to calculate connectivity [11], suffer from

16 lack of an additional constraint. This can be overcome by considering the

17 solid-phase structure of the material, e.g. the shape and size distribution of

18 mineral grains. The solid phase characteristics are incorporated in this work

19 to improve the realism of the constructed PNMs. The new method

20 developed in the work can be coupled directly to the existing lattice models

21 of the solid-phase previously developed for analysis of damage evolution

22 via micro-cracking [13, 14].

The first objective of the work is to develop a methodology for pore network construction for materials with partially available experimental data. The second objective is to develop the model to account for anion exclusion and sorption effects on the mass transport. The third objective is to validate 28 the method with experimental data, for which OPA is selected.

\section{2. Methodology}

\section{$30 \quad$ 2.1. Experimental data}

31 Opalinus Clay displays anisotropic responses to deformation and transport sedimentation and compaction [15]. Specifically, experiments indicated anisotropic diffusion of solute species with slow diffusion perpendicular and fast diffusion parallel to the bedding plane. Our goal is to construct a regular PNM in line with available structural data and macroscopic observations.

The pore space used in this work is OPA with sandy facies, labelled BDR in Keller et al. [9, 16], in which a large number of pores are located predominantly within the fine-grained clay mineral matrix. These pores with radii > $10 \mathrm{~nm}$, called meso-pores, were elongated in the bedding plane, which was resolved by Focused Ion Beam nano-tomography (FIB-nt). The 
1 hereafter micro-pores, occupied approximately 9.7 vol.\% (obtained from $\mathrm{N}_{2}$ 2 adsorption analysis). The porosity of micro-pores is thus $\theta_{\text {mic }}=0.097$.

3 Further, the meso-pores were largely isolated and did not provide a 4 percolating network through the sample.

The above two measurements were combined into a single 'cumulative pore 7 volume fraction - pore radius' curve given in Fig 1(a) [9, 11]. For model 8 construction the experimental distribution from Fig. 1(a) is re-evaluated as 9 cumulative probability separately for meso- and micro-pores. These are 10 shown in Fig. 1(b) and Fig. 1(c), respectively.

Regarding the solid phase, Keller et al. [17] reported 18 vol.\% non-porous carbonates with sizes ranging between $100 \mathrm{~nm}$ and $300 \mathrm{~nm}$ and 17 vol.\% of non-porous quartz, the size distribution of which was undetermined. For constructing the model for this study, the reported data was converted into cumulative probability of carbonate grain sizes as shown in Fig. 1(d). As 18 the size distribution of the carbonate particles due to lack of quartz-specific 19 experimental data.
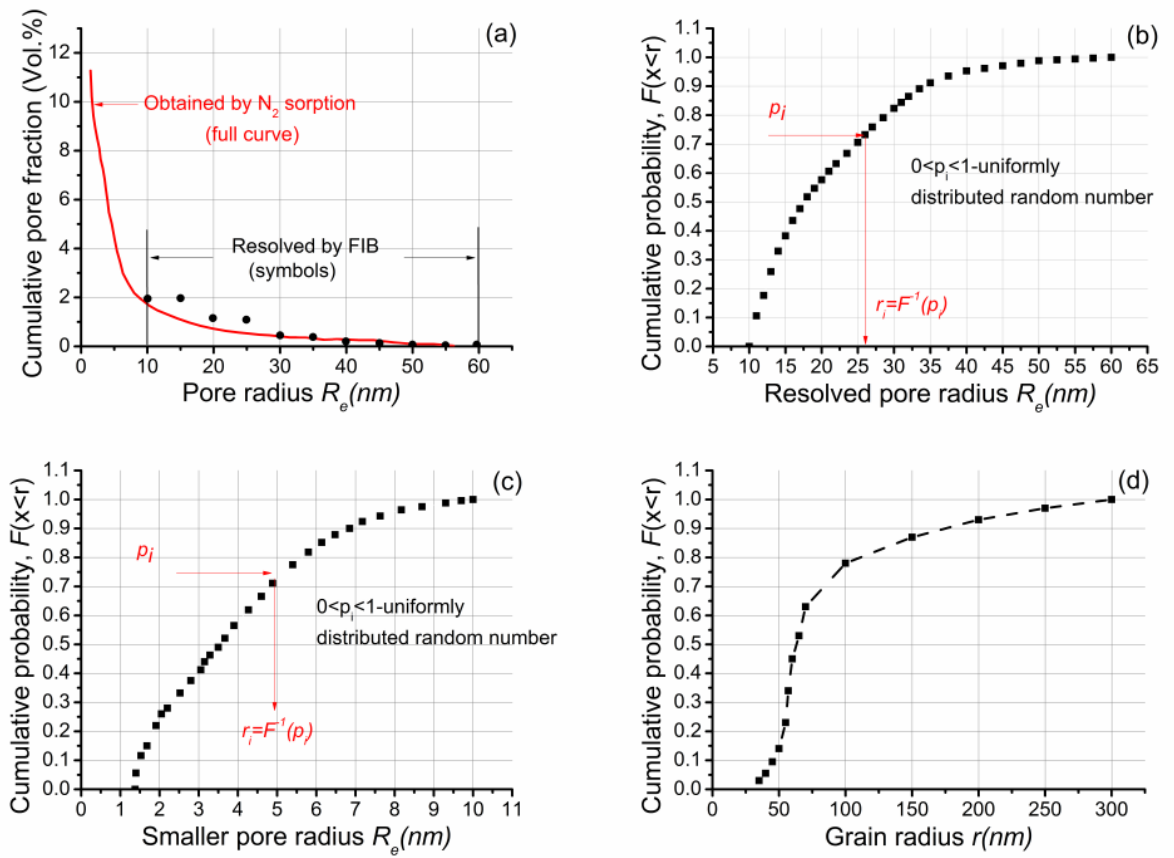

Fig. 1 Microstructure characteristics of Opalinus Clay: (a) cumulative pore volume fraction versus pore size determined by FIB-nt and $\mathrm{N}_{2}$ adsorption analyses of sample BDR [9]; (b) cumulative distribution of meso-pore sizes; (c) cumulative distribution of micro-pore sizes with assumed probability density [11]; (d) cumulative distribution of carbonate grain sizes [17]. 


\subsection{Pore network construction}

2 Many pore networks are constructed based on different length scales due to

3 the broad range of pore size distribution. A short overview of existing multi-

4 scale pore network models is presented here.

5

Jiang et al. [18] developed a methodology to integrate networks extracted from images at distinct length scales. The pore network model was generated at each scale and then was integrated into a single two-scale network by characterizing the cross-scale connection structure between the two networks. The shortcoming of this method is that it is computationally costly due to the number of network elements [19]. Recognizing the computational problems when single micro-pores are taken into account, Mehmani and Prodanović [20] proposed a two-scale pore network by packing algorithms. The macro-network is constructed by Delaunay tessellation of the grain centres. Micro-porous networks are generated by downscaling existing networks extracted from macro-pores. This approach was capable of investigating fundamental two-phase flow properties of multi-scale porous media and micro-porosity were able to act in series (intergranular or pore-filling micro-porosity) and in parallel (intra-granular or dissolution micro-porosity) to the macro-pores. However, in the construction process, distorted pores produced when many small grains touched a large grain. A parameter, the ratio of the macro to micro length scale, needs to be determined for micro-porous regions from the measurements which may be difficult to obtain. Boultry et al. [19] developed a workflow to integrate networks of macro-pores and microporous regions extracted from micro-CT images. This methodology allowed micro-porosity to act both in parallel and in series with the macro-pore network. However, a representative network for the micro-porosity is necessary. As the truncated cone shape is used to connect neighbouring two macro-pores, the tortuosity of the connection and geometric details about the bulk of the micro-porous cluster are neglected, which can lead to erroneous local conductivities. In this work, we use regular networks to generate models based on meso-porosity and micro-porosity.

The workflow is as follows: Firstly, we select the cellular basis for allocation of grains and pores, which results in complementary (dual) lattices for solid and pore systems. In this work, the material was subdivided into cells, in which the truncated octahedrons represented the neighbourhoods of particles in the OPA. The truncated octahedron was the unit cell of a regular space tessellation, proposed for site-bond modelling of solids [13], which was used successfully for mechanical analysis [14, 21] as well as in our previous works on transport problems $[8,11,12]$. 
1 The particles or grains are associated with cell centres (interiors). This is

2 illustrated in Fig. 2(a) for cells with equal distances between the three pairs

3 of square faces, a setup used in previous works $[11,12]$. The geometry of

4 the cellular assembly is described by three length parameters, $S_{1}, S_{2}$, and $S_{3}$,

5 measuring the distances between the square faces in directions $(1,0,0),(0$,

$61,0)$ and $(0,0,1)$, respectively. In an assembly of $N_{c}$ cells, the particle

7 radius, $r_{i}$ from Fig. 1(d), is assigned in each cell. The volume of all allocated

8 particles is required to be equal to the experimentally-measured particle

9 volume fraction, $\phi$. From this requirement the volume of a cell assembly is

10 calculated by:

$$
V_{c}=\frac{1}{\phi N_{c}} \sum_{i=1}^{N_{c}} \frac{4 \pi r_{i}^{3}}{3} \quad\left(=\frac{1}{2} S_{1} S_{2} S_{3}\right)
$$

12 where $V_{c}$ is the volume of a cell. The calculation of the three length 13 parameters from Eq. (1) depends on the selection of their ratios used here to 14 represent texture (a non-textured medium would have $S_{1}=S_{2}=S_{3}$ ).

Considering the fact that the reported pore radii are derived with the assumption of cylindrical pore shapes [9], we propose a new pore network structure. Firstly, a skeleton is formed using sites at the centres of cell faces and bonds between neighbouring faces. This is illustrated in Fig. 2(b) on a single cell with $S_{1}=S_{2}=S_{3}$. The bonds show the positions where elongated cylindrical pores are allowed to reside, i.e. they represent potential diffusion pathways. Since pores reside on bonds, the sites at face centres represent pore junctions - volume-less containers redirecting mass transport. Then the experimentally measured porosity is mapped to the proposed skeleton. The meso-pores are allocated along cell boundaries according to their size distribution, relative porosity and in preferred direction(s), while the micropores with radii selected from Fig. 1(c) are assigned to bonds not already occupied by the meso-pores. The process terminates when the total pore volume fraction attains the experimental porosity. An example of a pore network model assigning meso-pores and micro-pore sets is shown in Fig. 2(c) and Fig. 2(d). More details about meso- and micro-pore allocation can 32 be found in Jivkov and Xiong [11]. 

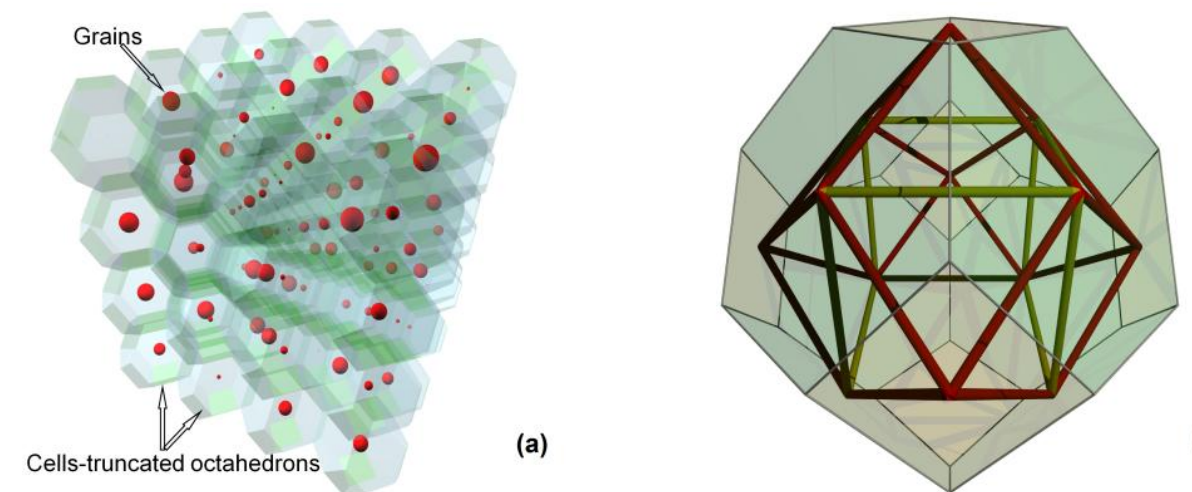

(b)
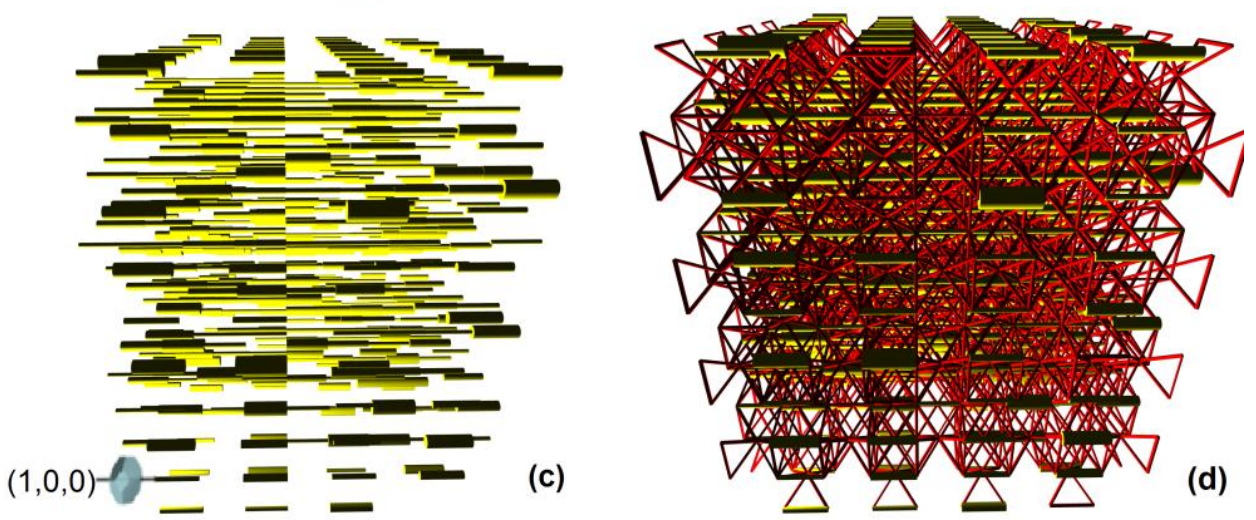

Fig. 2 (a) a cellular assembly, illustrating particles of variable sizes allocated to cell interiors; (b) unit cell illustrating potential diffusion paths (bonds) in the neighbourhood of central particle; (c) exclusive assignment of mesopores along $(1,0,0)$ taken as the clay bedding direction; (d) pore network model after assignment of meso-pores (yellow) and micro-pores (red).

9 Examples are given on lattice with equal length parameters for bedding and 10 out-of-bedding directions.

\section{2.3. Pore diffusivities}

13 Real pores and throats have complex and highly irregular geometrical 14 profiles. In this work, a dimensionless circularity $G$ was introduced to 15 approximate throats as cylindrical capillaries with a constant cross section:

$$
G=\frac{4 \pi A_{e}}{P^{2}}
$$

17 where $A_{e}=\pi R_{e}^{2}\left[\mathrm{~nm}^{2}\right]$ is the cross-section area, $R_{\mathrm{e}}=$ radius [nm] and $P=$ 18 perimeter $[\mathrm{nm}]$. The overall pore shape in the clay matrix parallel and 19 perpendicular to the bedding is elongated with a mean circularity of 0.45 20 [22].

22 The diffusion in the constructed model is driven by the concentration 23 gradient along the cylindrical pores. For a pore with radius $R_{e}$, connecting 
1 nodes $n$ and $m$, the mass flux of diffusing species, $J_{e}\left[\mathrm{~kg} / \mathrm{m}^{2} \mathrm{~s}\right]$, is described

2 by the Fick's first law:

$$
J_{e}=-D_{p} G \Delta C_{n m} / L_{e}
$$

4 where $D_{p}\left[\mathrm{~m}^{2} / \mathrm{s}\right]$ is the pore diffusivity, $L_{e}[\mathrm{~m}]$ is the pore length, and $\Delta C_{n m}=$ $5 C_{n}-C_{m}\left[\mathrm{~kg} / \mathrm{m}^{3}\right]$ is the concentration difference between nodes. The pore 6 diffusivity of species of radius $r_{0}$ is given by [23]:

$$
D_{p}=D_{0}\left(1-r_{0} / \mathrm{R}_{\mathrm{e}}\right)^{4}
$$

8 where $D_{0}$ is the free molecular diffusion coefficient of the species in the

9 medium filling the pore system. Pore diffusivities enter a graph-theoretical 10 representation of PNM, allowing for efficient solution of the transport 11 through the network via discrete analysis on graphs as detailed by Jivkov 12 and Xiong [11]. The mass flow though a pore, $W$, is then $W=J_{e} \times A_{e}$.

\section{2.4. Anion exclusion effects}

15 The effective diffusion coefficients, which are a function of the tortuosity 16 and constrictivity in the clay, and the accessible porosity, are different for 17 various solutes [24]. In OPA, diffusion of anions is reduced relative to a 18 neutral tracer such as tritiated water (HTO). The decreased diffusion is 19 explained by anion exclusion effects. The anion exclusion effects are caused 20 by the diffuse double layer (DDL) that surrounds the negatively charged clay surface. To calculate effective diffusion coefficients for anions, e.g. $\mathrm{Cl}$ and $\mathrm{Br}^{-}$, the amount of DDL needs to be modelled firstly. As the amount of DDL-water is assumed equal to half of the porosity which is not accessible for anions due to anion exclusion [25], the volume of DDL is achieved by increasing the layer thickness of DDL, $r_{t}$, in each pore. If $R_{e}<r_{t}$, the anions cannot pass through this pore, otherwise, the anion-accessible pore radius will be $R^{*}=\left(R_{e}-r_{t}\right)$.

In this work, we first model the diffusion of HTO to obtain the total porosity, and then, model the $\mathrm{Cl}^{-}$to find the anion-accessible porosity. The difference gives the amount of DDL-water which is assigned to be devoid of porosity. Iodide has the same accessible porosity as $\mathrm{Cl}^{-}$. However, the flux front for $I^{-}$is retarded compared with that of $\mathrm{Cl}^{-}$and $\mathrm{Br}^{-}$. A likely explanation of this retardation is the linear sorption of $I^{-}$onto the clay surface $[26,27]$.

\subsection{Sorption effects}

38 OPA has large specific surface area [26], high ion-exchange capacity [26], 39 and sorption affinity for ions, suggesting that long-term diffusivity of such 40 species can be significantly affected by sorption [28]. The amount of species 41 sorbed onto the solid, $\beta$, is described by the sorption isotherm, $\beta=\beta\left(C_{M}\right)$, 
1 where $C_{M}$ is the local concentration of species $\mathrm{M}$ in the solution. Different

2 species have linear or nonlinear sorption processes [29].

3

In order to simulate sorption effect, the sorption of species needs to be judged firstly. In this work, the species are assumed to be strongly adsorbed so that the estimate of the sorption obstruction effect can be obtained by considering the adsorbate to be uniformly smeared into a layer of thickness, $t$, as described in Xiong et al. [5, 12]:

$$
t^{2}-2 t R_{e}+8 \theta R_{e} r_{M} / 3=0
$$

where $r_{M}$ is the radius of the diffusing species. If $t<r_{M}$, the species should be weakly adsorbed. This means that the above sorption equation (Eq. 5) underestimates the sorption obstruction effect [12]. In this case, the sorption effects can be achieved by reducing the radii of throats by a layer of thickness $r_{M}$ [5]. The radius of a throat after sorption becomes $R^{*}=R_{e}-r_{M}$. The distribution coefficient $K_{d}$ of $I^{-}$is $0.02 \mathrm{~cm}^{3} / \mathrm{g}$, thus the linear sorption isotherm, $\beta$, equals $K_{d} C_{M}$ [30].

\section{Results and discussion}

With respect to a coordinate system $\left(X_{1}, X_{2}, X_{3}\right)$ normal to the square faces of the unit cell (see Fig. 2), a pore network skeleton within the boxed region $\left(0 \leq X_{1} \leq 20 S_{1}, 0 \leq X_{2} \leq 20 S_{2}, 0 \leq X_{3} \leq 20 S_{3}\right)$ was used. The size of the model is sufficiently large to reduce the variation of resulting diffusivity from an average under $10 \%$. The selection of the two boundaries depends on the macroscopic diffusivity being analysed. Specifically, the boundary conditions used to calculate the macroscopic diffusivity $D_{1}$ parallel to bedding direction $(1,0,0)$ are: prescribed concentration $C_{0}$ in all nodes on plane $X_{1}=0$; prescribed concentration $C_{1}$ in all nodes on plane $X_{1}=20 S_{1}$; zero flux through all nodes on planes $X_{2}=0, X_{2}=20 S_{2}, X_{3}=0, X_{3}=20 S_{3}$. For calculating the macroscopic diffusivity $D_{2}$ perpendicular to the bedding direction, say $(0,1,0)$, the boundary conditions are: prescribed concentration $C_{0}$ in all nodes on plane $X_{2}=0$; prescribed concentration $C_{1}$ in all nodes on plane $X_{2}=20 S_{2}$; zero flux through all nodes on planes $X_{1}=0$, $X_{1}=20 S_{1}, X_{3}=0, X_{3}=20 S_{3}$.

\subsection{Texture effect on diffusivity}

The pore networks constructed exhibit macroscopic tortuosity (path lengthening), introduced by the selection of diffusion pathways along the interfaces between solid-phase regions. This tortuosity depends on the material texture, represented here by the ratios of the cell length parameters in three perpendicular directions. Experimental results show that tortuosity is larger in a direction perpendicular to the bedding plane [31]. Therefore, 
1 we investigate the effect of larger cell length along the bedding plane and 2 smaller cell lengths perpendicular to the bedding plane.

3

The out-of-bedding directions are not differentiated, i.e. the case of having $S_{2}=S_{3}$ is assumed. Three ratios of the cell length parameters are considered: $S_{1} / S_{2}=2,1.5,1$. For each cellular assembly of given ratio, 10 realisations of pore spatial distributions were analysed to obtain the diffusion coefficients along the bedding plane, $D_{1}$, and out-of-bedding directions, $D_{2}$. The results reported in Fig. 3 are the averaged values of these analyses on the basis of containing $35 \mathrm{vol} \%$ solid particles. The calculated diffusion coefficients of HTO are in the following ranges: $D_{1}=3.09 \times 10^{-11} \sim 5.73 \times 10^{-11}$ $\mathrm{m}^{2} / \mathrm{s} ; \quad D_{2}=1.42 \times 10^{-11} \sim 2.46 \times 10^{-11} \mathrm{~m}^{2} / \mathrm{s}$ (see Fig. 3). Reported experimentally obtained values for HTO diffusion in OPA shown in Fig. 3 are $D_{1}=(5.4 \pm 0.4) \times 10^{-11} \mathrm{~m}^{2} / \mathrm{s}[29,31]$ and $D_{2}=1.13 \times 10^{-11} \sim 1.55 \times 10^{-11}$ $\mathrm{m}^{2} / \mathrm{s} \quad[30,32]$. When $S_{1} / S_{2}=2$, the simulated results are closest to the experimental values. This ratio is used to calculate diffusivities for anions in the following section. The difference between the computational and the experimental results could be partially due to a difference between the microstructure characteristics of OPA obtained by Keller et al. [9, 16] and used for model construction, and the clays used for experimental measurement of effective diffusion coefficients [30, 32].

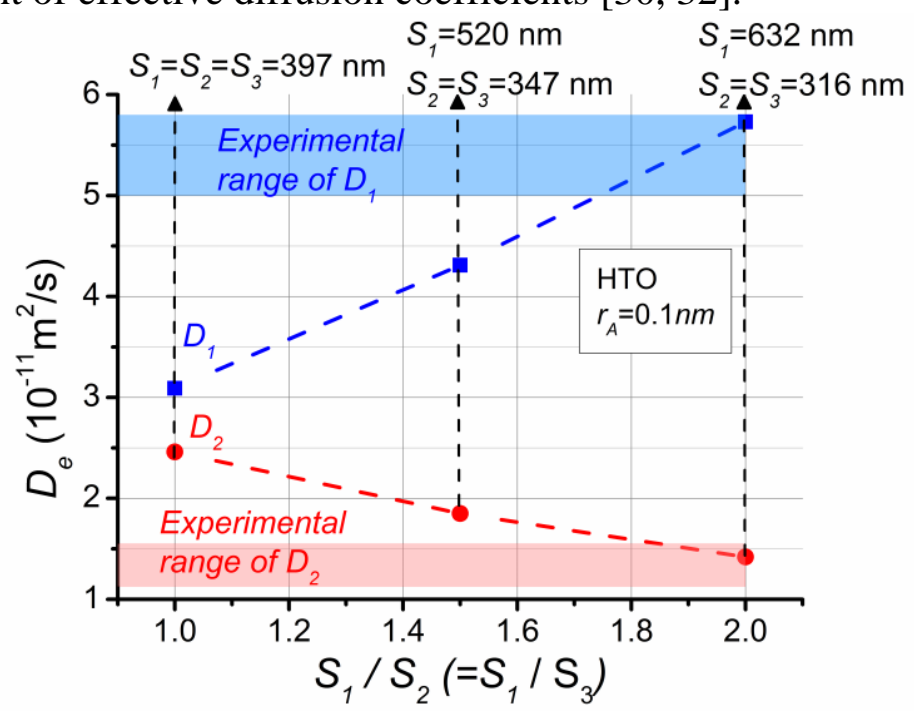

Fig. 3. Calculated macroscopic (effective) diffusivity of HTO in OPA. Clay textures are measured by the ratio of length parameters in the bedding, $S_{1}$, and the out-of-bedding, $S_{2}$, directions. $D_{1}$ and $D_{2}$ are the diffusion coefficients parallel and perpendicular to the bedding plane.

\section{3.2. Anion exclusion and sorption effect on diffusivity}

29 The macroscopic diffusivities of $\mathrm{Cl}^{-}$and $I$ are shown in Table 1. The

30 simulated results of $\mathrm{Cl}^{-}$are very close to the experimental effective 
diffusivities [30]. The predicted $D_{1}$ of anions is a little overestimated and $D_{2}$ of anions is underestimated. This is in agreement with the expectations for

3 the following reasons. Firstly, allocation of $r_{t}$ leads to larger number of 4 micro-pores belonging to DDL and a higher increase of macroscopic 5 tortuosity in out-of-bedding directions. Since pores allocated in out-of6 bedding directions are only micro-pores and the layer thickness of DDL, $r_{t}$, 7 assigned in each pore is the same. A larger fraction of micro-pores is not 8 accessible for the anions in out-of-bedding direction compared to the 9 number of pores in bedding plane direction. As a result, the effective 10 diffusivities in out-of-bedding directions are under estimated. Because the total DDL volume is 50 vol.\% of the pores and the micro-pores in out-ofbedding directions contribute more to the DDL volume, the effective diffusivities in the bedding plane direction will be over estimated. Secondly, the accessible porosity for anions should be in a small range [29, 30]. This model can be used to calculate other anion effective diffusivities which only have anion exclusion effects.

The diffusion behaviour of $I$ is the effect of anion exclusion and sorption. Thus, the sorption is simulated on the basis of the same accessible porosity as of $\mathrm{Cl}^{-}$. The predicted effective diffusivities of $I$ are in agreement with measured values as shown in Table 1. The over/under estimation of experimental value, $D_{1}$ and $D_{2}$, is mainly due to the same reason (exclusion

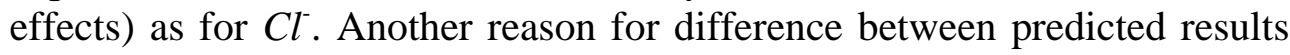
and experimental values could be that the sorption isotherm measured by through-diffusion technique [30] is not quite accurate to describe sorption onto pore walls.

Table1. Comparison of experimental (EXP) and computational (COM) results for diffusion of different species: $D_{0}=$ free molecular diffusion coefficient; $D_{1}=$ diffusivity in OPA bedding direction; $D_{2}=$ diffusivity in OPA out-of-bedding direction. All diffusion coefficients are scaled by $10^{-12}$ $\mathrm{m}^{2} / \mathrm{s}$.

\begin{tabular}{|c|c|c|c|l|c|}
\hline \multirow{2}{*}{ Species } & $D_{0}$ & \multicolumn{2}{|c|}{$D_{1}$} & \multicolumn{2}{c|}{$D_{2}$} \\
\cline { 3 - 6 } & & EXP & MOD & EXP & MOD \\
\hline$C l^{-}$ & 2030 & $15 \sim 17$ & 18 & $5.31 \sim 5.77$ & 3.82 \\
\hline$I$ & 2000 & $10 \sim 14$ & 16 & $4.28 \sim 4.86$ & 3.36 \\
\hline
\end{tabular}

33 Note: The free molecular diffusion coefficients $D_{0}$ at room temperature are

34 obtained from Mont Terri Project [29]. Experimental effective diffusivities 35 are obtained from Van Loon, LR [30].

\section{4. Conclusions}

37 This work presented:

- A new method for pore network construction, where solid-phase characteristics of a porous media are used to balance incomplete 


\section{References}

41

1. Yu, J.-W. and I. Neretnieks, Diffusion and sorption properties of radionuclides in compacted bentonite. 1997: Svensk 
2. Bourg, I.C., A.C.M. Bourg, and G. Sposito, Modeling diffusion and 4 adsorption in compacted bentonite: a critical review. Journal of

3. Grathwohl, P., Diffusion in natural porous media: contaminant Contaminant Hydrology, 2003. 61(1-4): p. 293-302.

transport, sorption/desorption and dissolution kinetics (POD). 1998.

4. Whitaker, S., Diffusion and dispersion in porous media. AIChE Journal, 1967. 13(3): p. 420-427.

5. Meyers, J. and A. Liapis, Network modeling of the convective flow and diffusion of molecules adsorbing in monoliths and in porous particles packed in a chromatographic column. Journal of Chromatography A, 1999. 852(1): p. 3-23.

6. Dillard, L.A. and M.J. Blunt, Development of a pore network simulation model to study nonaqueous phase liquid dissolution. Water Resources Research, 2000. 36(2): p. 439-454.

7. Gao, S., J.N. Meegoda, and L. Hu, Two methods for pore network of porous media. International Journal for Numerical and Analytical Methods in Geomechanics, 2012. 36(18): p. 1954-1970.

8. Jivkov, A.P., et al., A novel architecture for pore network modelling with applications to permeability of porous media. Journal of Hydrology, 2013. 486: p. 246-258.

9. Keller, L.M., et al., 3D geometry and topology of pore pathways in Opalinus Clay: Implications for mass transport. Applied Clay Science, 2011. 52(1-2): p. 85-95.

10. NAGRA, Projekt Opalinuston: Synthese der geowissenschaftlichen Untersuchungsergebnisse Technischer Bericht 2002. 02 -03: p. 250252.

11. Jivkov, A.P. and Q. Xiong, A Network Model for Diffusion in Media with Partially Resolvable Pore Space Characteristics. Transport in Porous Media, 2014. 105(1): p. 83-104.

12. Xiong, Q., A.P. Jivkov, and J.R. Yates, Discrete modelling of contaminant diffusion in porous media with sorption. Microporous and Mesoporous Materials, 2014. 185: p. 51-60.

13. Jivkov, A.P. and J.R. Yates, Elastic behaviour of a regular lattice for meso-scale modelling of solids. International Journal of Solids and Structures, 2012. 49(22): p. 3089-3099.

14. Zhang, M. and A. Jivkov, Microstructure-informed modelling of damage evolution in cement paste using a site-bond model. Construction and Building Materials, 2014. 66: p. 731-742. 
15. Wenk, H.-R., et al., Preferred orientations and anisotropy in shales: Callovo-Oxfordian shale (France) and Opalinus Clay (Switzerland). Clays and clay minerals, 2008. 56(3): p. 285-306.

16. Keller, L.M., et al., Pore space relevant for gas permeability in Opalinus Clay: Statistical analysis of homogeneity, percolation, and representative volume element. Journal of Geophysical Research: Solid Earth, 2013. 118(6): p. 2799-2812.

17. Keller, L.M., et al., Characterization of multi-scale microstructural features in Opalinus Clay. Microporous and Mesoporous Materials, 2013. 170: p. 83-94.

18. Jiang, Z., et al., Representation of multiscale heterogeneity via multiscale pore networks. Water resources research, 2013. 49(9): $p$. 5437-5449.

19. Bultreys, T., L. Van Hoorebeke, and V. Cnudde, Multi-scale, microcomputed tomography-based pore network models to simulate drainage in heterogeneous rocks. Advances in Water Resources, 2015. 78(0): p. 36-49.

20. Mehmani, A. and M. Prodanović, The effect of microporosity on transport properties in porous media. Advances in Water Resources, 2014. 63: p. 104-119.

21. Jivkov, A.P., M. Gunther, and K.P. Travis, Site-bond modelling of porous quasi-brittle media. Mineralogical Magazine, 2012. 76(8): p. 2969-2974.

22. Houben, M.E., G. Desbois, and J.L. Urai, Pore morphology and distribution in the Shaly facies of Opalinus Clay (Mont Terri, Switzerland): Insights from representative 2D BIB-SEM investigations on $\mathrm{mm}$ to $\mathrm{nm}$ scale. Applied Clay Science, 2013. 71: $\mathrm{p}$. 82-97.

23. Bryntesson, L.M., Pore network modelling of the behaviour of a solute in chromatography media: transient and steady-state diffusion properties. Journal of Chromatography A, 2002. 945(1): p. 103-115.

24. Appelo, C.A.J. and D. Postma, Geochemistry, groundwater and pollution. 2005: CRC Press.

25. Appelo, C.A.J. and P. Wersin, Multicomponent diffusion modeling in clay systems with application to the diffusion of tritium, iodide, and sodium in Opalinus Clay. Environmental science \& technology, 2007. 41(14): p. 5002-5007.

26. Altmann, S., et al., Diffusion-driven transport in clayrock formations. Applied Geochemistry, 2012. 27(2): p. 463-478. 
1 27. Appelo, C., L. Van Loon, and P. Wersin, Multicomponent diffusion of a suite of tracers (HTO, Cl, Br, I, Na, Sr, Cs) in a single sample of Opalinus Clay. Geochimica et Cosmochimica Acta, 2010. 74(4): p. 1201-1219.

28. Aytas, S., M. Yurtlu, and R. Donat, Adsorption characteristic of U(VI) ion onto thermally activated bentonite. J Hazard Mater, 2009. 172(2-3): p. 667-74.

29. Project, M.T., Long-term Diffusion (DI-A) Experiment: DI-A2: Diffusion of $\mathrm{HTO}, \mathrm{Br}^{-}, \mathrm{I}^{-} \mathrm{Cs}^{+},{ }^{85} \mathrm{Sr}^{2+}$ and ${ }^{60} \mathrm{Co}^{2+}$ : Field activities, data and modelling. Report TR, 2010: p. 2009-04.

30. Van Loon, L., J. Soler, and M. Bradbury, Diffusion of $\mathrm{HTO}^{36} \mathrm{Cl}^{-}$and ${ }^{125} I^{-}$in Opalinus Clay samples from Mont Terri: Effect of confining pressure. Journal of Contaminant Hydrology, 2003. 61(1): p. 73-83.

31. Van Loon, L.R., et al., Anisotropic diffusion in layered argillaceous rocks: a case study with Opalinus Clay. Environmental science \& technology, 2004. 38(21): p. 5721-5728.

32. Joseph, C., et al., Diffusion of U(VI) in Opalinus Clay: Influence of temperature and humic acid. Geochimica et Cosmochimica Acta, 2013. 109: p. 74-89.

33. Sok, R.M., et al., Direct and stochastic generation of network models from tomographic images; effect of topology on residual saturations. Transport in porous media, 2002. 46(2-3): p. 345-371. 